\section{INTRAOPERATIVE ULTRASONIC CHOLANGIOGRAPHY FOR BILIARY SYSTEM IDENTIFICATION}

We read with great interest the paper "Intraoperative ultrasonography of the biliary tract using saline as a contrast agent: a fast and accurate technique to identify complex biliary anatomy" by Chandra and colleagues. ${ }^{1}$ They reported that saline sonocholangiography (SSC) provided excellent images for correct identification of segmental bile duct anatomy during surgery of the biliary tract, and was a simple and inexpensive technique that could be performed with minimal resources.

We recently developed contrastenhanced intraoperative ultrasonic cholangiography (CE-IOUSC) using perfluorobutane microbubbles (Sonazoid; Daiichi-Sankyo Pharmaceutical Co., Japan) as a tool for real-time biliary navigation in various hepatobiliary operations. ${ }^{2-4}$ This technique enables 3-dimensional mapping and visualization of the 2-dimensional regional anatomy of the biliary tree. ${ }^{2}$ In addition, we reported that CE-IOUSC could delineate the biliary drainage areas of the liver parenchyma belonging to a bile duct orifice with a clear dividing line as a pseudostaining region. ${ }^{3}$ We think that CE-IOUSC and SSC are similar in concept but use different contrast agents, although both contrast agents consist of saline and bubbles. We would like to point out several concerns.

We feel that concentration and persistence time of the contrast agent are key factors for success of ultrasonic cholangiography imaging and real-time biliary navigation. At higher concentrations of the contrast agent, acoustic shadowing induced by condensed microbubbles in bile ducts interferes with ultrasound imaging. At lower concentrations, the echogenicity of bile ducts is reduced, preventing their vis- ualization against a background of hyperechoic surrounding tissues, such as those in the hilar plate. In the study by Chandra and colleagues, a high concentration of saline bubbles in the bile duct cast a sonic shadow that interfered with imaging of structures in the liver beyond the bile duct. We emphasize that CE-IOUSC can be performed using a stable contrast agent concentration because dilution is performed in the same manner as for intrabiliary injection, compared with use of saline bubbles made of churned air, which are too unstable for optimal concentration. The short contrast agent persistence time requires frequent injections for real-time anatomic identification of the biliary tree. In addition, the short persistence time interferes with imaging of smaller bile duct branches, such as the caudate branches. In fact, the authors reported that echogenicity lasted for only for 1-2 minutes or less in some patients. This duration is too short for real-time biliary navigation. This is why we selected perfluorobutane microbubbles as a contrast agent for CE-IOUSC. We agree that saline bubbles are less expensive, but speculate that bubbles made from saline and air churning lack important characteristics adequate for use as a contrast agent for CE-IOUSC.

Lastly, we emphasize the importance of performing hepatobiliary operations without complications. We hope that novel intraoperative ultrasonic cholangiography techniques without radiation exposure, such as CE-IOUSC or SSC, will be widely used to reduce biliary complications during hepatobiliary operations.

\section{Takeshi Urade, MD, PhD; Takumi Fukumoto, MD, PhD}

Affiliations: From the Department of Surgery, Division of Hepato-Biliary-Pancreatic Surgery, Kobe University Graduate School of Medicine, Kobe, Japan (Urade, Fukumoto); and the Department of Surgery and Digestive Surgery, Kita-Harima Medical Center, Ono, Japan (Urade).
Competing interests: None declared.

DOI: $10.1503 /$ cjs. 1861011

\section{References}

1. Chandra A, Gupta V, Rahul R, et al. Intraoperative ultrasonography of the biliary tract using saline as a contrast agent: a fast and accurate technique to identify complex biliary anatomy. Can 7 Surg 2017;60:316-22.

2. Urade T, Fukumoto T, Tanaka M, et al. Contrast-enhanced intraoperative ultrasonic cholangiography for real-time biliary navigation in hepatobiliary surgery. 7 Am Coll Surg 2014;218:e43-50.

3. Fukumoto T, Urade T, Kido M, et al. A novel technique for the intraoperative identification of biliary drainage areas in the liver after hepatobiliary resection for perihilar cholangiocarcinoma. $7 \mathrm{Am}$ Coll Surg 2016;222:e31-8.

4. Urade T, Fukumoto T, Kido M, et al. Contrast-enhanced intraoperative ultrasonic cholangiography in living donor hepatectomy. Liver Transpl 2016;22:1437-42.

\section{AUTHOR RESPONSE}

We thank Drs. Urade and Fukumoto for their comments on our paper. Urade and colleagues described contrast-enhanced intraoperative ultrasonic cholangiography for real-time biliary navigation using perfluorobutane microbubbles in their 2014 study, referenced in our paper. As they mentioned, one limitation of their study was the use of a specific contrast agent and $3 \mathrm{D}$ ultrasound probes, which may not be available across all centres, as in our case. We studied the feasibility of using saline, churned with room air as a contrast agent along with a $2 \mathrm{D}$ ultrasound probe as an alternative. As mentioned by Drs. Urade and Fukumoto, achieving a specific concentration and stability of saline bubbles was not possible in our study, and this also explains variations in the amount of echogenicity and contrast retention time seen on our ultrasound images. These variations are also mentioned in our results. Constant saline injection in the 